\title{
Template-Based Sampling of Anisotropic BRDFs
}

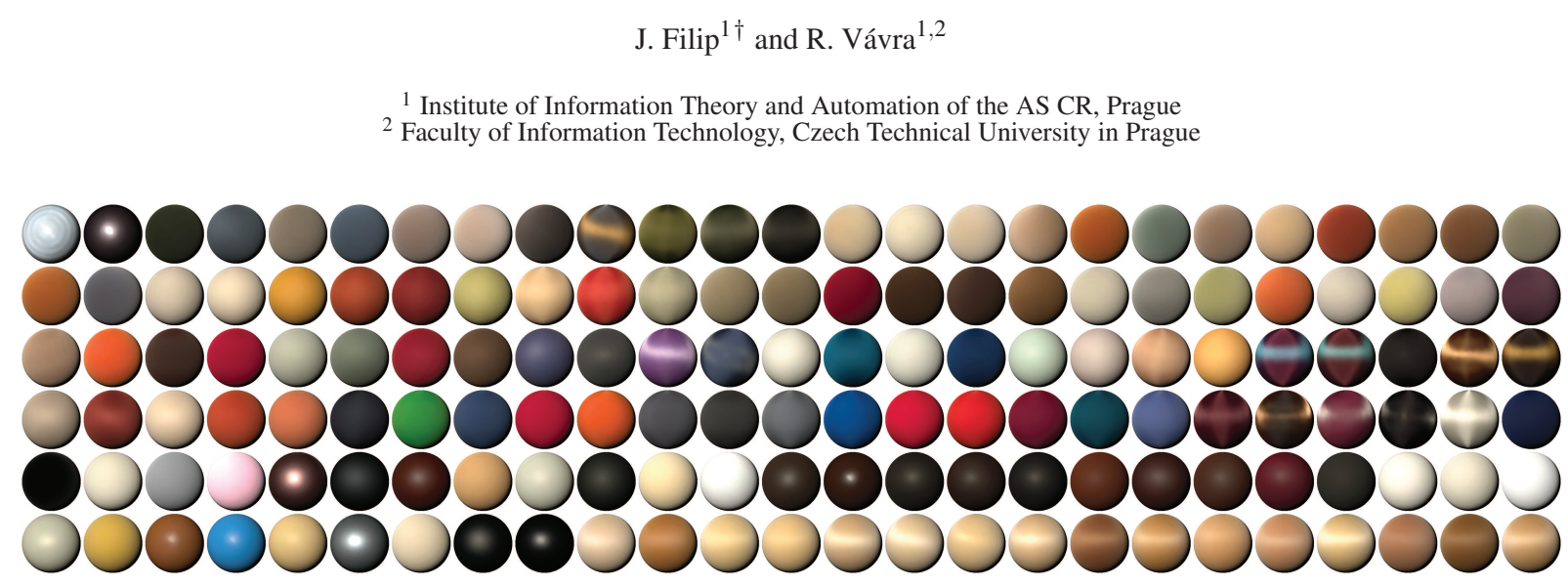

Figure 1: Renderings of 150 materials recorded in the proposed BRDF database.

\begin{abstract}
BRDFs are commonly used to represent given materials' appearance in computer graphics and related fields. Although, in the recent past, BRDFs have been extensively measured, compressed, and fitted by a variety of analytical models, most research has been primarily focused on simplified isotropic BRDFs. In this paper, we present a unique database of $150 \mathrm{BRDF}$ sepresenting a wide range of materials; the majority exhibiting anisotropic behavior. Since time-consuming BRDF measurement represents a major obstacle in the digital material appearance reproduction pipeline, we tested several approaches estimating a very limited set of samples capable of high quality appearance reconstruction. Initially, we aligned all measured BRDFs according to the location of the anisotropic highlights. Then we propose an adaptive sampling method based on analysis of the measured BRDFs. For each $B R D F$, a unique sampling pattern was computed, given a predefined count of samples. Further, template-based methods are introduced based on reusing of the precomputed sampling patterns. This approach enables a more efficient measurement of unknown BRDFs while preserving the visual fidelity for the majority of tested materials. Our method exhibits better performance and stability than competing sparse sampling approaches; especially for higher numbers of samples.
\end{abstract}

Categories and Subject Descriptors (according to ACM CCS): I.3.7 [Computer Graphics]: Three-Dimensional Graphics and Realism-Color, shading, shadowing, and texture I.3.4 [Computer Graphics]: Digitization and Image Capture-Reflectance

\section{Introduction}

Bidirectional reflectance distribution function (BRDF) has been widely used to represent appearance of real-world materials in computer graphics applications. A monospectral $\operatorname{BRDF}\left(\theta_{i}, \varphi_{i}, \theta_{v}, \varphi_{v}\right)$ is a four dimensional function of incoming $\omega_{i}=\left[\theta_{i}, \varphi_{i}\right]$ and outgoing $\omega_{v}=\left[\theta_{v}, \varphi_{v}\right]$ spherical

\footnotetext{
† jiri.filip@utia.cas.cz
}

angles ( $\theta$ elevation, $\varphi$ azimuth) as shown in Fig. 2-a. A four-dimensional BRDF was formalized in [NRH*77] as a specific case of eight-dimensional Bidirectional ScatteringSurface Reflectance Distribution Function (BSSRDF), restricted due to its properties [DF97], to flat and opaque materials. Accurate BRDF measurement is challenging due to a huge number of combinations of various illumination and viewing angles; therefore, the majority of BRDF measurement and analysis approaches have focused on simplified isotropic BRDFs. By neglecting reflectance change, due to 
the rotation of the sample around its normal, for fixed illumination and viewing angles one can obtain only a threedimensional isotropic BRDF function $\operatorname{BRDF}\left(\theta_{i}, \theta_{v}, \varphi_{i}-\right.$ $\left.\varphi_{v}\right)$. A practical usage of BRDF, is due to missing affordable and reliable measurement limited to fitting analytical models to sparsely measured directions. While this approach suppresses noise in the data, it however, cannot retain all the features of material angularly dependent behavior. Although accurate anisotropic BRDF datasets are needed, the related long acquisition times limit their availability. In our work we propose a novel BRDF database and focus on adaptive acquisition of BRDF achieving a better reconstruction accuracy than using comparable fixed sampling techniques.

Therefore, our main contributions are:

- Introduction of a new BRDF database containing 150 measurements of wide range of materials. Contrary, to majority of the past research we deal with full fourdimensional anisotropic BRDF measurements.

- Analysis of the BRDFs anisotropic behavior and their main anisotropy axis alignment.

- An estimation of a proper directional sampling of each BRDF given the predefined number of samples and introduction of template-based method reusing it for more efficient BRDF measurement,

- Thorough comparison of the presented method with alternative sparse sampling approaches.

The paper structure: Section 2 overviews previous research related to the BRDF measurement and sampling. Section 3 describes our BRDF acquisition process, Section 4 introduces our BRDF database and its properties, and Section 5 describes preprocessing and anisotropy axis alignment procedures. Section 6 explains our approximation of ideal reference sampling, while Section 7 proposes two templatebased material dependent sampling approaches. Section 8 compares these approaches with competing methods and Section 9 concludes the paper.

\section{Related Work}

As our work contributes to several specific areas, we will discuss related work separately in three categories.

BRDF measurement - Although BRDF represents a basic building block of material appearance representation in computer graphics, due to a complex measurement process, to date reasonable quality datasets are quite rare. BRDFs have been primarily measured by gonioreflectometers, i.e., devices independently positioning a light source and sensor over a measurement hemisphere [HLZ10,ELU11]. Since such a process has been time consuming, faster mirrorand image-based setups have been developed to allow the recording of more illumination/viewing directions simultaneously. Mirror-based setups allow more rapid measurement using hemispherical [War92], elliptic [Dan01, GAHO07], or parabolic mirrors [MSY07], at the cost of a lower range of possible elevation angles or dynamics of the measured data. Conversely, image-based setups [MPBM03a,
MWLT00, NDM05] rely on the known geometry of a convex object (sphere, cylinder) and use this information for appropriate binning of BRDF values from the illuminated object's recoded images. Also, other approaches are possible. A setup based on the combination of moving light on circular rail and sample orientation by a robotic arm has been introduced for industrial paint pigment measurements [HAH*12]. Approximate BRDF measurements can be obtained using either condenser reflectometry recording many views at once [LDW*10], or using a proprietary target with known BRDFs [RWS*11]. Another image-based approach estimates objects BRDF from single view and illumination [SHGW13] by extraction and compensation of sparse BRDF data which are later fitted by an analytical model. Although the approximative measurement setups allow fast data acquisition, they are restricted to isotropic BRDFs only.

BRDF databases - Despite the number of published BRDF measurement approaches being relatively high, there are very few measurements available to the research community at large. Since the well-known MERL database of Matusik et al. comprising one hundred isotropic BRDFs was published in 2003 [MPBM03a], only much later smaller numbers of samples were presented in other research projects. BRDFs of eight automotive paints were introduced in $\left[\mathrm{GCG}^{*} 05\right]$ and BRDFs of four paints, skin, ceramic, and felt fabric were published in [MWLT00]. Ngan et al. [NDM05] performed fitting of all MERL datasets from database by several analytical models. Also, they provided four additional anisotropic BRDF measurements using their own image-based setup and made them available. As most measurement processes suffer heavily from noise; consequently, there is a lack of accurate dense BRDF data for research purposes. Alternative measurements of four anisotropic materials in high angular density were presented in [FVH].

In this paper a new BRDF database measured using a goniometric setup is presented. Most of the samples show anisotropic behavior; and, although their angular density is not as high as in [NDM05, FVH], we believe that the dataset constitute a notable contribution relative to Matusik's dataset. Its main limitation was given by isotropy and measured specimen preparation. Image-based approaches require placing the sample on the surface of a sphere/cylinder. Challenges result because some flexible materials would exhibit unwanted folds when wrapped, and is incompatible for use with rigid materials such as woods or tiles. Alternatively, amongst other materials, our datasets contain BRDFs of fabric, wood, leather, plastic and the like not publicly available from any other source.

BRDF adaptive measurement attempts reconstruction of unknown BRDF using data-driven placement of new samples. The first category of such approaches estimate a fixed set of directional samples using a linear combination of BRDFs from a database or their compression into a common basis and iterative search for a well-conditioned subset 
of the required number of measurement directions. Then, for any new material only, this subset of several hundred samples has to be measured, and the respective BRDF is obtained by linear combination of BRDFs or basis-vectors using estimated coefficients. Matusik et al. [MPBM03b] represented MERL database using wavelet-basis and the linear combination of BRDFs. Later on Ali et al. [ASOS12] used 2D bivariate representation to improve representation by linear basis. Although there are approaches to direct BRDF measurement in this representation from a single image given the know illumination environment [RVZ08], they are limited to a certain subset of isotropic materials only.

The second category, performs a stepwise data-driven measurement of an unknown BRDF function. Such appearance measurement was investigated in [FBLS07], yet only in two dimensions representing sampling of the reflectance field. An adaptive approach of image-based BRDF measurement was proposed in [LLSS03] with planning view and illumination directions based on uncertainty minimization of analytical model parameters. Although one can use global interpolation/error prediction methods [PTVF92], unfortunately, they have high computational demands which become intractable when the number of samples exceed several thousand. Although standard empirical or physical analytical BRDF models provide often unsatisfactory fitting of BRDFs, a recent research moves toward generating a more efficient, material-class dependent analytical isotropic BRDF models using genetic programing [BLPW14]. Recently, an approach to anisotropic BRDF acquisition using perpendicular adaptively sampled slices in BRDF subspaces has been proposed in [FVH*13].

In this paper we compared the uniform, linear combination-based [MPBM03b], linear basisbased [ASOS12] sampling estimation techniques, and the adaptive BRDF slices [FVH*13] with a two proposed variants of template-based sampling method relying on the closest sampling pattern selection from a set of materials recorded in our BRDF database.

\section{A BRDF Acquisition Setup}

All data were measured using a gonioreflectometric device introduced in $\left[\mathrm{FVH}^{*} 13\right]$. The device is based on two independently controlled arms containing custom-build LED light (surface illuminance $12000 \mathrm{lux}$ ) and industrial fullframe 16Mpix RGB camera AVT Pike 1600C (their distance from sample $1.1 \mathrm{~m}, 2.1 \mathrm{~m}$, arms positioning accuracy $0.03^{\circ}$ ), recording reflectance of sample on rotating platform placed below the arms. Rotation axes of both arms and the platform intersect at the measured material's surface. All BRDF samples are recorded in HDR typically using five combinations of lighting intensity and camera exposure relative to measured material reflectivity. Performed prior to each measurement was radiometric and colorimetric calibration. Uniform angular density of $81 \times 81=6561$ directions [SSK03] in our BRDFs is relatively sparse, however, for the majority of the materials it is sufficient to capture their behav- ior as the highlights are not too narrow. Measurements at all elevations have the same level of reliability. All images from different views/illuminations were mutually registered and rectified first. Then the representative area of real size $11 \times 11 \mathrm{~mm}$ was cropped and its values spatially averaged. One of the advantages of spatial averaging in BRDF acquisition is the suppression of noise in the data. An example of a typical mean measurement noise can be observed by two measurements of the same corduroy fabric specimen. When we computed a difference between their captured raw measured BRDFs (prior to $N \cdot L$ compensation and reciprocity enforcement) we obtained PSNR 79.03. All BRDFs were recorded as float values and 4-dimensions of anisotropic BRDF were stored as 2D images divided into azimuthally dependent BRDF subspaces for fixed elevation angles. Because the measurement over six thousand directions takes around nine hours we performed batch measurements of nine materials simultaneously, each spanning the region of $3 \times 3 \mathrm{~cm}$, where varying angles across patches were compensated. As we recorded all combinations of illumination and viewing directions, we compensated for illuminated area foreshortening relative to increasing elevation and finally enforced reciprocity which helped to suppress possible sources of noise in the measurement process. Each of the BRDFs

(a)

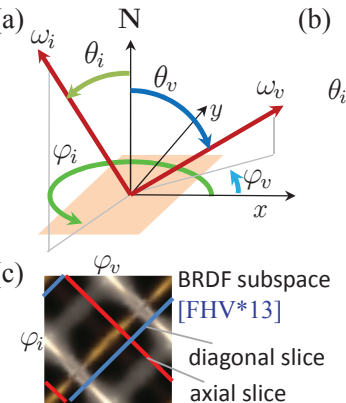

(b)

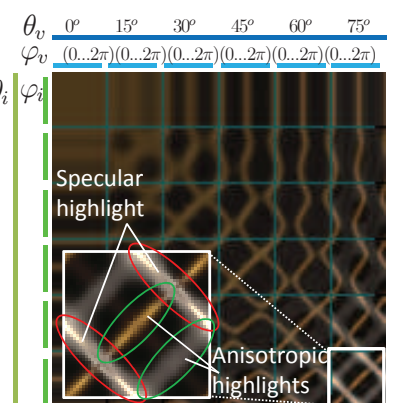

Figure 2: (a) BRDF local coordinate space, (b) BRDF of fabric112 with specular and anisotropic highlights in a BRDF subspace, and (c) BRDF slices in a subspace.

measurements for $81 \times 81$ hemispherical uniform sampling, resulted in a variable count of samples at different elevation angles. In order to obtain the fixed count of samples needed for our further analysis, we converted these 6561 samples to angularly uniform directions. As we have six elevation angles we obtain $6 \times 48 \times 6 \times 48=288 \times 288=82944$ directions (see Fig. 2-b). We used an interpolation based on radial basis function [PTVF92].Details of individual materials are shown in supplementary material. All raw and interpolated measured BRDFs are available in binary and OpenEXR formats $^{\dagger}$ and physical samples may be provided up on request.

$\dagger$ http://btf.utia.cas.cz 


\section{A BRDF Database}

We recorded 150 BRDFs in total (see Fig. 1). In addition to a majority of fabrics, we measured leather, plastic, wood, paper, plaster, etc. As seen in Tab. 1, we focused on anisotropic materials, that can be categorized into groups and summarized according to strength of anisotropy, number of anisotropic highlights, and presence of two distinct colors. We aimed at collecting as many

Table 1: A categorization of measured materials indicating anisotropy strength, number of anisotropic highlights $(H L)$, and presence of two distinct colors in BRDF.

\begin{tabular}{l|r|rr|rr|l}
$\begin{array}{l}\text { material } \\
\text { category }\end{array}$ & total & \multicolumn{2}{|c}{ anisotropy } & \multicolumn{2}{c}{ anis. HLs } & \multicolumn{2}{c}{ two } \\
mat. & strong & weak & one & two & colors \\
\hline fabric & $\mathbf{9 6}$ & 21 & 51 & 41 & 31 & 6 \\
wood & $\mathbf{1 6}$ & 16 & - & 16 & - & - \\
leather & $\mathbf{1 6}$ & - & 2 & 2 & - & - \\
carpet & $\mathbf{6}$ & - & 2 & 2 & - & - \\
plastic & $\mathbf{6}$ & - & 1 & 1 & - & - \\
paper & $\mathbf{2}$ & - & - & - & - & - \\
wallpaper & $\mathbf{2}$ & - & - & - & - & - \\
plaster & $\mathbf{2}$ & - & - & - & - & - \\
others & $\mathbf{4}$ & - & 1 & 1 & - & - \\
\hline total & $\mathbf{1 5 0}$ & 37 & 57 & 63 & 31 & 6
\end{tabular}

types of anisotropic behaviors as possible. To achieve high variability we collected BRDFs of nearly homogeneous samples of leather, plastic, smooth fabric. Naturally, fabric materials present the highest variability of appearance due to individual thread-based weaving patterns; therefore, they constitute the biggest group of the measured materials. We avoided materials with macroscopic features. Most materials exhibit anisotropy caused by their smallscale geometry being recorded as high-resolution images.

A comparison of three fabric materials photographs with measured BRDF renderings arranged on a cylinder illuminated from above the camera is shown in Fig. 3.
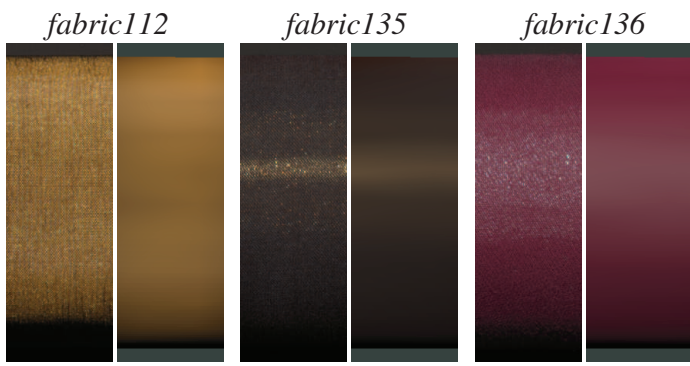

Figure 3: A Comparison of three measured materials (left) and their BRDF renderings (right) on a cylinder.

\section{BRDF Data Preprocessing}

This section describes initial BRDFs preprocessing and anisotropy alignment necessary for our further analysis.

BRDF Uniform Resampling and Interpolation - As the number of measured samples is insufficient for a proper sampling of more specular BRDFs, we performed interpolation of each subspace to the resolution of $48 \times 48$ directions (angular resolution $7.5^{\circ}$ ). In contrast to the MERL database [MPBM03a], the number of specular materials in our database is limited, therefore, we could afford a significantly lower sampling density. To improve the continuity of highlights we detected specular and anisotropic highlight positions in each subspace and performed linear interpolation just over values along the highlights. Obtained specular data were then added to measured samples and used for final interpolation based on two dimensional radial basis functions [PTVF92]. As a result, we obtained smoother specular and anisotropic highlights instead of discontinuous ones as shown in Fig. 4. Although

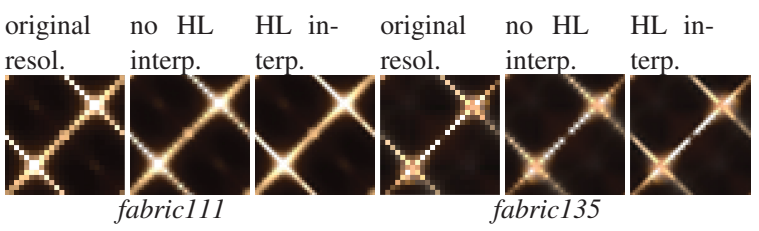

Figure 4: Two BRDFs interpolation - effect of specular and anisotropic highlights $(H L)$ linear interpolation.

the interpolation artificially modified raw BRDF measurements, it allowed us to obtain a database of relatively dense measurements for our continued analysis. We believe that our modifications are physically reasoned as the original measurements are altered to preserve a typical smooth specular highlights behavior present in our measurements.

Following the interpolation each measured BRDF is finally represented by $1+5 \times 48+(5 \times 48 \times 5 \times 48) / 2+$ $(5 \times 48) / 2=29161$ reciprocal samples $n_{s}$.

Anisotropic Highlight Alignment - Contrary to analysis of isotropic BRDFs, where locations of specular highlights can be predicted, the analysis of anisotropic ones is more challenging. The number and location of anisotropic highlights in angular space is unknown and thus depends entirely on the initial orientation of the measured material and on its properties. Therefore, before different anisotropic BRDFs can be compared, first they must be aligned according to the main anisotropic axis.

Wang et al. [WZT* 08 ] solved a related problem of normal distribution functions alignment and merging corresponding to different surface locations of the same material. In contrast to this approach we tackle a more general problem of BRDF alignment of materials having completely different types of anisotropic behavior.

The procedure is straightforward when we operate in a subspace of azimuthal angles (see Fig. 5).

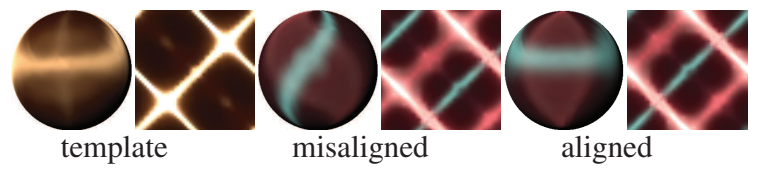

Figure 5: Example subspace (elevations $75^{\circ} / 75^{\circ}$ ) anisotropy alignment of material fabric107 according to template defined by material fabric 111 . 
As the subspace is circularly symmetric its circular shift (function circshift in MATLAB) in diagonal direction will have the same effect as the change of initial rotation of the measured sample prior to the measurement. As the correct detection of the main anisotropic highlight is crucial for our BRDF sampling experiments we tested several approaches. The best results were obtained by an alignment approach using a shiny anisotropic material (fabric111) with distinct specular and anisotropic highlights as a template $T$. A subspace of the query material $B$ is aligned using shift $\hat{s}$ to this template by minimizing $\mathrm{L} 1$ norm between the query and template subspaces, across all possible circular shifts of matrix $B$

$$
\hat{s}=\underset{s=0 . . \pi}{\arg \min } \sum_{\varphi_{i}=0}^{2 \pi} \sum_{\varphi_{v}=0}^{2 \pi}\left|T\left(\varphi_{i}, \varphi_{v}\right)-B\left(\operatorname{circshift}\left(\varphi_{i}, \varphi_{v},-s\right)\right)\right|
$$

Reconstruction Performance Evaluation between the reference and interpolated BRDFs $(B, \hat{B})$ in $n_{s}$ sample points is performed using peak signal-to-noise ratio (PSNR):

$$
P S N R=10 \cdot \log _{10}\left(\frac{n_{S}}{\sum_{i=1}^{n_{s}}[B(i)-\hat{B}(i)]^{2}}\right) .
$$

\section{Approximation of Optimal Sampling}

For the sake of comparing of the tested sparse sampling methods, we look for reference adaptive BRDF sampling for the required number of samples. We build on a heuristic algorithm introduced by Robinson and Ren [RR95] refining samples positions based on a cross-validation principle, and propose an Algorithm 1 that iteratively adds and shifts BRDF samples while minimizing error between the reconstructed and ground truth BRDF.

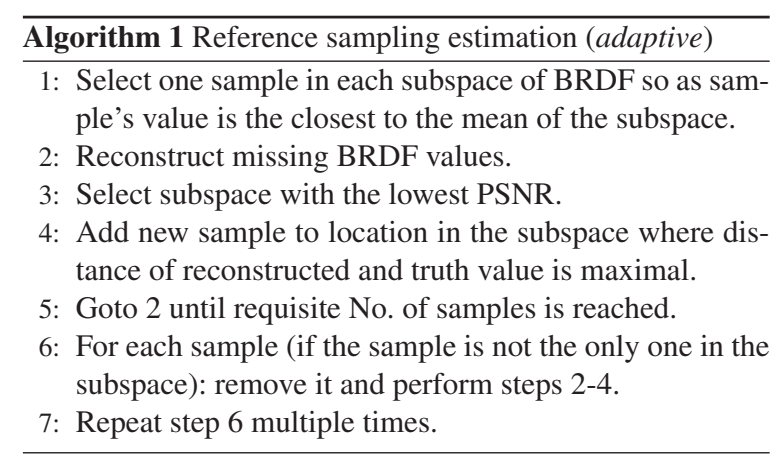

Note that this approach (denoted as adaptive) does not guarantee finding an optimal sampling, and some of the tested sampling techniques have shown even better performance; however, we believe that it can serve as a reasonable approximation of optimal sampling. We use thin-plate splines for data interpolation from the sparse samples. The reconstruction error from samples estimated using this algorithm is shown as a blue line in Fig. 6. Estimated samples for all BRDFs are shown in the supplemental material.
We also used Algorithm 1 for derivation of a fixed sampling pattern approximating entire BRDF database (denoted as global), i.e., we use all 150 BRDFs instead of using a single BRDF for adaptive samples placement.

\section{Proposed Template-Based Sampling}

This section estimates the proper angular sampling of unknown BRDFs, given a predefined number of samples, using a template-based sampling method. The method is based on reusing of precomputed sampling patterns (Algorithm 1) for a more efficient BRDF measurement of unknown material.

In contrast to the method introduced in the previous section, the sampling of unknown material is more difficult because we do not have a prior knowledge of material's angular behavior. Therefore, at least minimal information must be collected, prior to the selection of a sampling strategy. Main anisotropy axis alignment for an un-

\begin{tabular}{l} 
Algorithm 2 Template-based method (proposed1) \\
\hline 1: Record additional 42 samples adaptively along four \\
slices perpendicular to specular highlights in a single re- \\
ciprocal subspace at illumination/view elevations $60^{\circ}$. \\
2: Find the most similar (L1 norm) material $\mathcal{M}$ from \\
database based on 90 samples already taken. \\
3: Measure BRDF using the pattern of the material $\mathcal{M}$. \\
\hline
\end{tabular}

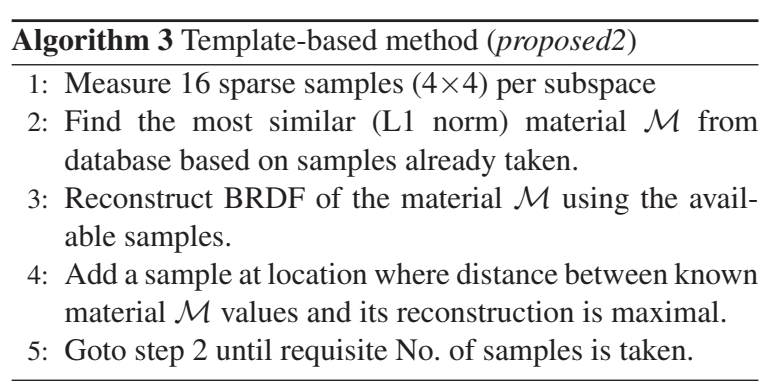

known material requires the initial measurement of 48 samples along the slice perpendicular to the anisotropic highlight (see an axial slice in Fig. 2-c). This number of samples, stemming from azimuthal resolution of our measurements, is then subtracted from the available count of samples in all tested sampling methods. Once material anisotropy is aligned, we look for proper precomputed sampling patterns obtained for each BRDF from the database using the method proposed in Section 6. We select the pattern that fits best to our sparse measurements using 90 samples. These measurements rely on the concept of BRDF slices [FVH*13] (see Fig. 2-c), that can be recorded by continuous azimuthal movements of light and camera. The entire sampling process (denoted as proposed l) is described in Algorithm 2. Note that the best results were achieved at subspace with elevations $60^{\circ}$, however, using different oblique elevations does not significantly impact the performance. 
Alternatively, we tested also the method's variant (denoted as proposed 2 ) that iteratively adds new samples and can change the chosen similar material if there is another more similar one based in the samples collected so far as described in Algorithm 3.

Both approaches directly compare values of unknown measured material $L_{U}$ with values of our BRDFs $L_{D}$ using L1 norm in locations of samples already taken. Values $L$ are obtained as weighted average of RGB values. To take into account different luminances of compared samples, we use least squares regression to identify linear parameters $a, k$ between vectors $L_{U}$ and $L_{D}$. The query material is then compared with all $N=150$ recorded BRDFs to find the one most resembling the sparse measurements using

$$
\hat{i}=\underset{i=1 \ldots N}{\arg \min }\left|L_{U}-\left(a_{i}+k_{i} \cdot L_{D i}\right)\right| .
$$

\section{Comparison of the Sampling Methods}

This section compares the proposed reference adaptive sampling (adaptive) with other sparse sampling approaches for a fixed number of samples.

Tested approaches overview - The compared techniques can be split in two categories. The first one comprises methods estimating fixed sampling pattern valid for any BRDFs: uniform sampling (uniform), linear combination (lin.comb.), and global adaptive sampling (global). The second one includes methods estimating sampling pattern of each measured material individually: BRDF slices (slices) and the template-based methods (proposed1, proposed2). The uniform sampling places samples to each BRDF subspace by means of regular matrices with resolution tuned independently in a way to achieve the lowest PSNR across the entire database for required count of samples (green line). The linear combination-based [MPBM03b] and linear basis-based [ASOS12] sampling estimation techniques gave nearly similar results, therefore, due to lower computational demands we selected linear combination-based results represented by magenta line. The global adaptive sampling is shown in brown line. Results of BRDF slices [FVH*13] (using 6 axial and 6 diagonal adaptively sampled slices per BRDF subspace) are shown in black line and results of the proposed baseline template-based method (proposed1) are illustrated in red. Its adaptive variant (proposed2) is represented in cyan. Methods global, proposed1, proposed2, and lin.comb. allocate the first 48 samples for anisotropic axis alignment of the BRDF using method presented in Section 5. Results of method proposed 1 and proposed 2 were computed with the query material excluded from the database. Similarly, the query material should be excluded from computation when sampling using lin.comb. and global methods, but computation times would be too long. Our experiments on several materials has shown that the impact of not excluding the query material is negligible.
Results - We performed experiments for different numbers of samples (as shown in a supplemental material); results for 1438 samples and all 150 BRDFs are shown in Fig. 6. The BRDFs are sorted according to the PSNR error of our reference adaptive sampling method (blue line). From the graph it is apparent that none of the tested methods is ideal for sparse sampling of all tested BRDFs. As expected, adaptive methods show better performance than fixed sampling-based methods. Their estimated sampling patterns for uniform, global, and lin.comb. methods are shown in the supplemental material. For BRDFs where the reference adaptive method obtained the worst PSNR (left side of the graph) worked the best the lin.comb. method while for the materials that can be represented in higher PSNR dominated the slices. Although lin.comb. approach shows superior performance for some of the difficult materials it has the worst stability across the BRDFs and is unable achieve better results than $\approx 45 \mathrm{~dB}$ (regardless the samples count). The template-based methods proposed 1 and proposed 2 marked steady and the most stable performance across all materials. Some of the tested methods indicate superior performance for some BRDFs yet perform poorly on some others but still keeping the same reconstruction PSNR as in more stable approaches. Therefore, performance instability (IS) was approximated by a standard deviation of the difference between the PSNR of adaptive sampling and of the methods tested. A legend of Fig. 6 shows PSNR values and performance instability averaged across all BRDFs.

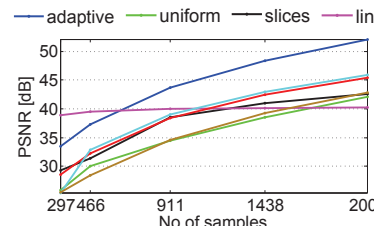

(a)

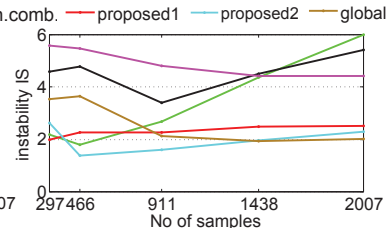

(b)
Figure 7: Reconstruction PSNR (a) and instability IS (b) of the compared methods as a function of a number of samples.

We also analyzed performance of all methods for variable number of samples. The reference adaptive sampling as well as sparse sampling using all the tested methods was computed for 297, 466, 911, 1438, and 2007 samples. The PSNR averaged across all samples and performance instability as a function of a number of samples are shown in Fig. 7.

In Fig. 7-a we can observe almost constant reconstruction performance of lin.comb. method (magenta line) regardless the number of samples. They are superior for material appearance representations using very small set of samples (under 1000), however, they are limited to a certain quality (e.g., $40 \mathrm{~dB}$ ) due to a limited descriptive power of a finite number of linear bases. When more than 1000-1500 samples is used all other techniques perform better. The highest reconstruction quality can be achieved using the proposed template-based sampling approaches (red, cyan), while worst results are achieved using uniform and global approaches (green and brown lines). 


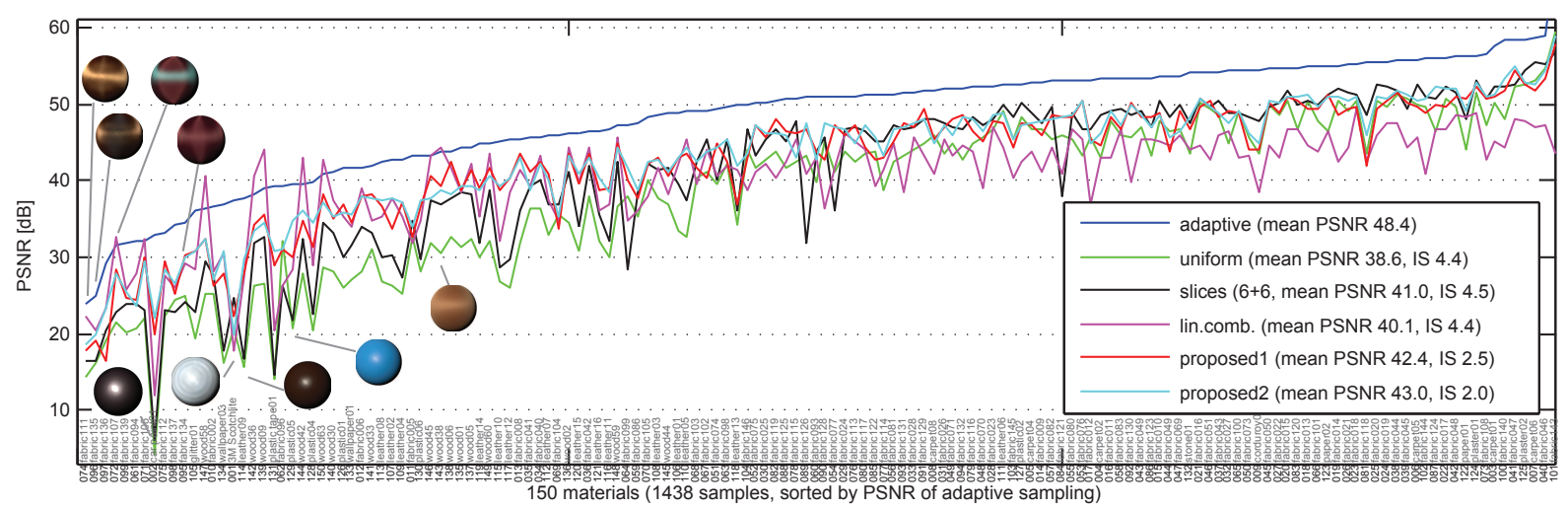

Figure 6: Comparison of reconstruction fidelity using fixed number of 1438 samples for all 150 BRDFs using estimated reference sampling (blue) and the tested sparse sampling approaches.

The lowest performance instability of the tested methods in Fig. 7-b are obtained using the proposed template-based techniques having an almost constant dependability across different number of samples. This is in contrast to the other tested approaches that mark higher or more variable performance instability with increasing numbers of samples.

Finally, Fig. 8 and Fig. 9 show example renderings for challenging nearly isotropic and anisotropic selected BRDFs from our database. We fixed a number of samples to 1438 and compared renderings obtained by all the tested approaches, with reference BRDF data and reference adaptive sampling. The $10 \times$ scaled difference images reveal that uniform and global approaches perform the worst, while lin.comb. generally works well at the cost of increased color error especially for materials whose representation using limited basis is difficult. Similarly, slices performed very well with exception of the grazing elevation angles. Performance of the proposed methods is well balanced across BRDFs and elevation angles.

Timings - The proposed adaptive and template-based sampling techniques are suboptimal approaches only. To find an optimal BRDF sampling pattern would be extremely computationally-demanding. Computational times for 1438 samples were as follows: adaptive took 70 minutes for each BRDF (global 140 minutes), while finding closest sampling for an unknown material using the approach proposed look 2 seconds and proposed 2 around 2 minutes using a single core of an Intel Xeon E5-2643. Finding the fixed pattern using lin.comb. by means of 33 iterations took one day, and slices selected sampling for each BRDF in 8 seconds. Of course, these times would increase if measurement process were to be included, but we believe that these times can be absorbed by the data acquisition times often exceeding several hours.

Discussion and Limitations - We also tested hard limits of the template-based methods by evaluation of all sampling patterns for reconstruction of the query material's BRDF and selecting the one with the lowest error. This test has shown that overall performance gain is mere $\approx 1 d B$, and thus proved that the proposed adaptive pattern selection approaches are correct without much room for improvement. An advantage of the fixed sampling pattern methods is universal sampling applicable for any measured materials albeit at the cost of lower reconstruction quality. An advantage of the method proposed 1 over its variant proposed 2 and slices is that it does not require any on the fly data analysis during the measurement. Main factors affecting error in our sampling pattern selection process were possibly the limited scope of our BRDF dataset (some materials of unique reflectance were presented only once) and number/distribution of initial sparse samples.

\section{Conclusions}

Presented is a publicly available database of 150 BRDFs, which, to our best knowledge, is the first extensive database focusing on anisotropic behavior of various materials.

As the measurement of BRDF is time demanding, we compared several sparse sampling BRDF approaches. First, we introduced a reference adaptive sparse sampling algorithm. This heuristic algorithm adaptively places the required number of directional measurements to find suboptimal reference angular sampling patterns. Then, we have introduced a template-based sampling algorithm selecting a proper sampling pattern for unknown materials based on 90 sparse samples only. The second proposed method allows pattern change during the course of the measurement to achieve better results. We compared our templatebased sparse sampling techniques with uniform sampling, linear combination of BRDFs, and BRDF slices approaches.

Our cross-validation experiments over the entire database has shown that none of the tested methods performed perfectly across all BRDFs from our database. If less than a thousand samples is needed or a lower reconstruction error can be afforded, the method linearly combining measured BRDFs works the best. In all other cases, the methods based on BRDF slices or on the proposed template-based matching dominate. The best stability of reconstruction performance across tested BRDFs was achieved by the template-based method. 


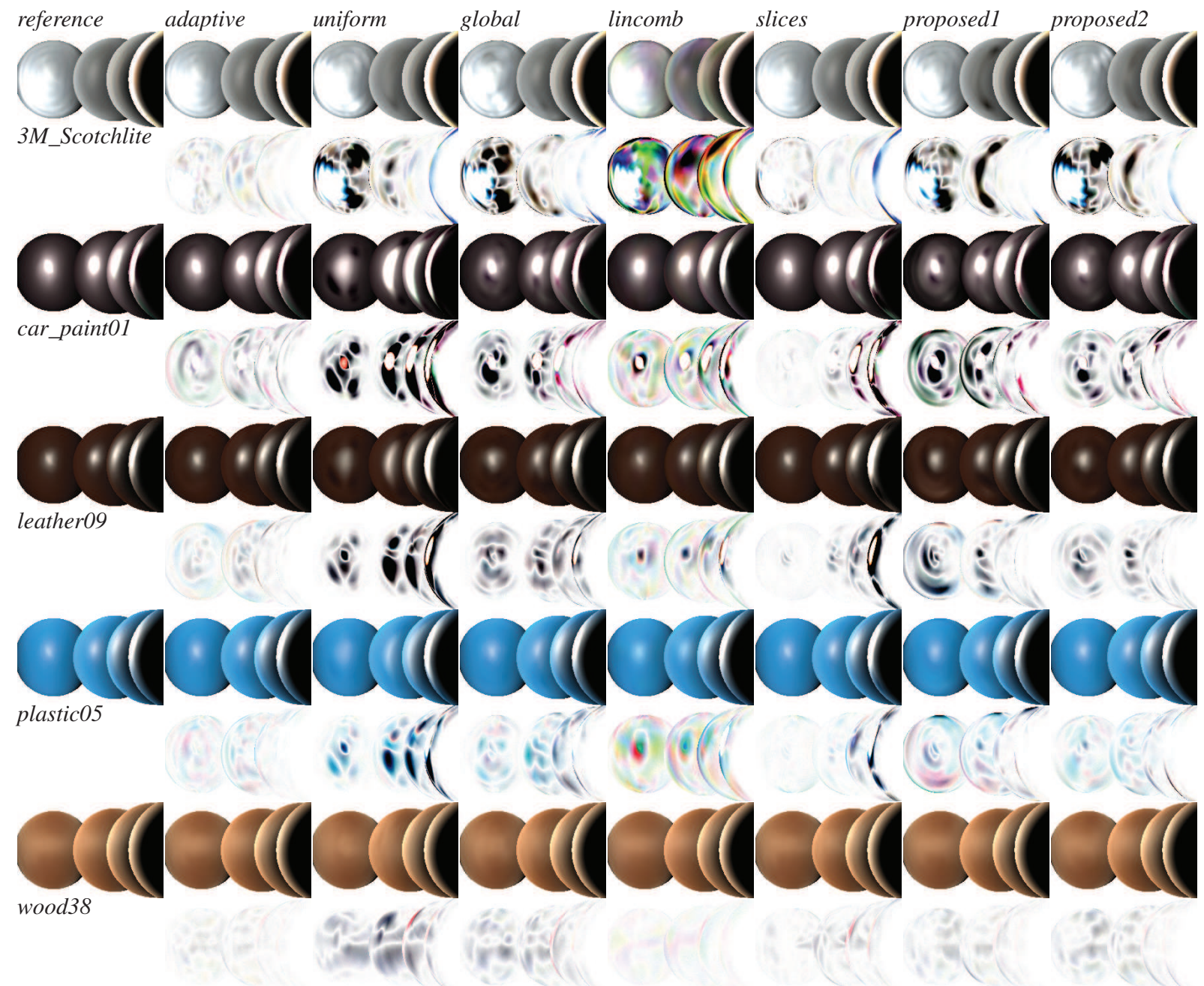

Figure 8: Rendering of four nearly isotropic and one anisotropic BRDFs on four spheres: reference vs. their reconstruction using 1438 samples obtained by the seven tested methods (columns). The difference images in even rows are scaled $10 \times$.

We believe that our data-driven sparse sampling study has shown that anisotropic BRDFs represent challenging data and that the current adaptive approaches are able to achieve good reconstruction quality using fewer than 2000 samples.

\section{Acknowledgments}

This research has been supported by the Czech Science Foundation grants 103/11/0335, 14-02652S, and 14-10911S.

\section{References}

[ASOS12] Ali M. A., Sato I., OKabe T., Sato Y.: Toward efficient acquisition of BRDFs with fewer samples. In ACCV (2012), pp. 54-67. 3, 6

[BlPW14] Brady A., Lawrence J., Peers P., Weimer W.: genBRDF: Discovering new analytic BRDFs with genetic programming. ACM Trans. Graph. 33, 4 (2014), 114:1-114:11. 3

[Dan01] DANA K.: BRDF/BTF measurement device. In Proceedings of ICCV 2001 (2001), vol. 2, pp. 460-466. 2
[DF97] DeYoung J., Fournier A.: Properties of tabulated bidirectional reflectance distribution functions. In Proc. of the Graphics Interface 1997, May 21-23 (1997), pp. 47-55. 1

[ELU11] Eilertsen G., LARSSON P., Unger J.: A versatile material reflectance measurement system for use in production. In SIGRAD 2011 (2011). 2

[FBLS07] FuChS M., BlanZ V., LENSCH H. P., SEIDEL H.-P. Adaptive sampling of reflectance fields. ACM Trans. Graph. 26 (June 2007), 1-18. 3

[FVH] FILIP J., VAVRA R., HAVLICEK M.: Effective acquistion of dense anisotropic BRDF. In ICPR 2014. 2

[FVH*13] FiliP J., VAVRA R., HAINDL M., Zid P., KRUPICKA M., HAVRAN V.: BRDF slices: Accurate adaptive anisotropic appearance acquisition. In $C V P R$ (2013), pp. 4321-4326. 3, 5, 6

[GAHO07] Ghosh A., Achutha S., Heidrich W., O'Toole M.: BRDF acquisition with basis illumination. IEEE ICCV (2007), 1-8. 2

[GCG*05] GÜnther J., Chen T., Goesele M., Wald I., SEIDEL H.-P.: Efficient acquisition and realistic rendering of car paint. In $V M V$ (2005), pp. 487-494. 2 


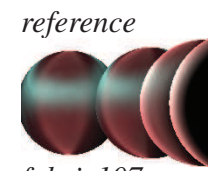

fabric 107

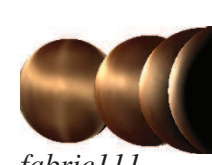

fabric 111

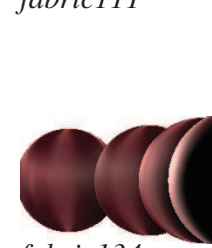

fabric 134

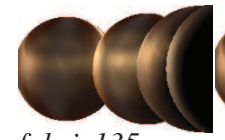

fabric135
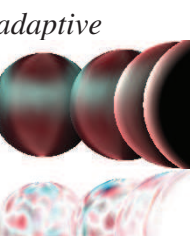

$a^{2} \lim ^{2}$
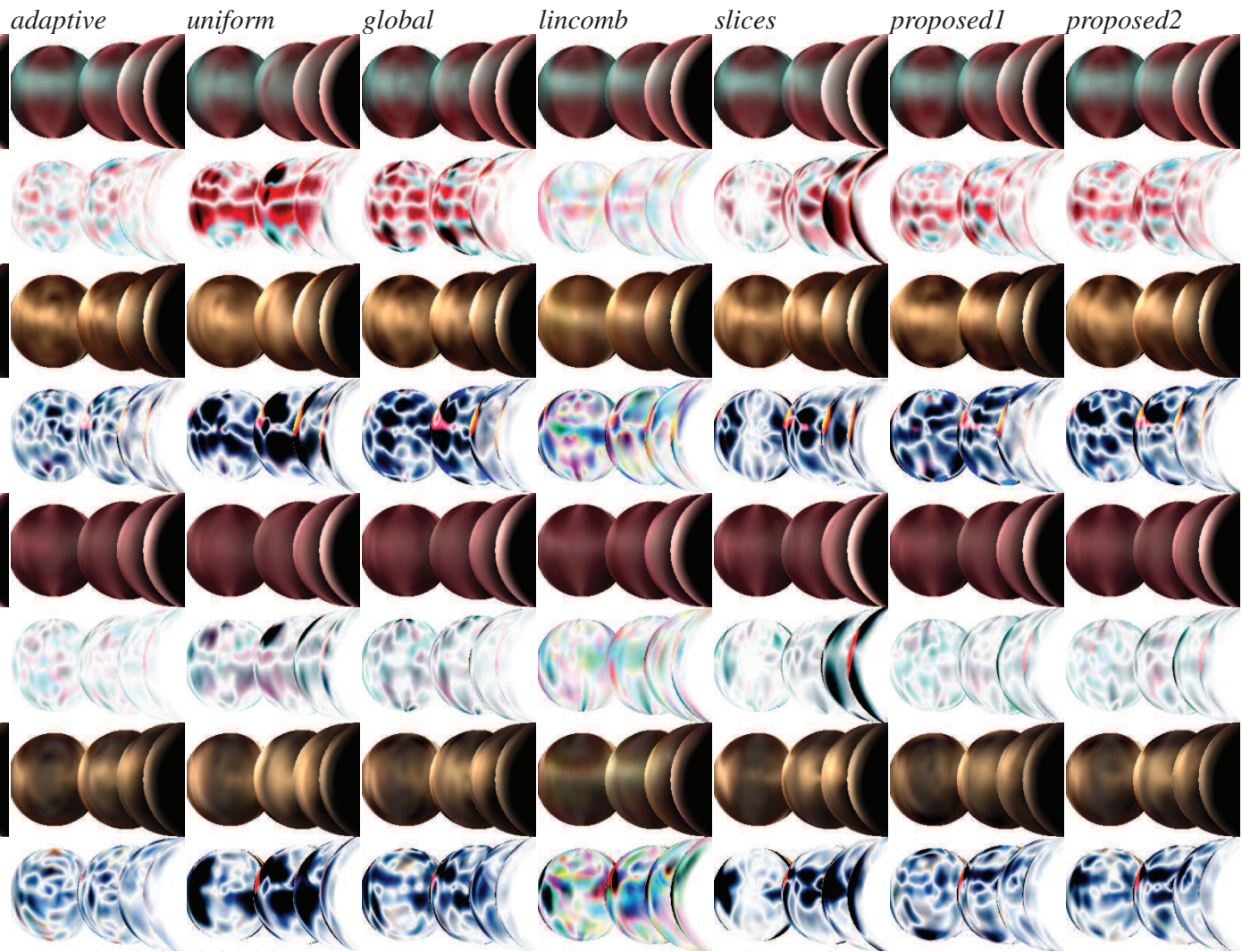
using 1438 samples obtained by the seven tested methods (columns). The difference images in even rows are scaled $10 \times$.

[HAH*12] Höpe A., Atamas T., Hünerhoff D., Teichert S., HAUER K.-O.: Argon ${ }^{3}$ : 3D appearance robot-based gonioreflectometer at PTB. Review of Scientific Instr. 83, 4 (2012). 2

[HLZ10] Holroyd M., LAWRenCE J., Zickler T.: A coaxial optical scanner for synchronous acquisition of 3D geometry and surface reflectance. ACM Trans. Graph. 29 (2010), 99:1-99:12.

[LDW*10] LAN Y., Dong Y., Wang J., TONG X., GuO B.: Condenser-based instant reflectometry. Computer Graphics Forum 29, 7 (2010), 2091-2098. 2

[LlSS03] Lensch H. P., LANG J., SÁ A. M., Seidel H.-P.: Planned sampling of spatially varying BRDFs. Computer Graphics Forum 22, 3 (2003), 473-482. 3

[MPBM03a] Matusik W., Pfister H., Brand M., McMilLAN L.: A data-driven reflectance model. ACM Transactions on Graphics 22, 3 (2003), 759-769. 2, 4

[MPBM03b] Matusik W., Pfister H., Brand M., McMilLAN L.: Efficient isotropic BRDF measurement. In Proceedings of the 14th Eurographics Workshop on Rendering (2003), EGRW '03, pp. 241-247. 3, 6

[MSY07] MUKaIGawa Y, SUmino K., Yagi Y: Multiplexed illumination for measuring BRDF using an ellipsoidal mirror and a projector. In ACCV'07 - Volume Part II (2007), ACCV'07, pp. 246-257. 2

[MWLT00] MARSCHNER S. R., WeSTIN S. H., LAFORTUNE E. P. F., TORRANCE K. E.: Image-based bidirectional reflectance distribution function measurement. Applied Optics 39 (2000). 2

[NDM05] NGan A., Durand F., MATusiK W.: Experimental analysis of BRDF models. Eurographics Symposium on Rendering 20052 (2005), 117-126. 2

[NRH*77] NicOdEMUS F., RichmOND J., HSIA J., GinsBURG I., LIMPERIS T.: Geometrical considerations and nomenclature for reflectance. NBS Monograph 160 (1977), 1-52. 1

[PTVF92] Press W. H., Teukolsky S. A., Vetterling W. T., Flannery B. P.: Numerical Recipes in C: The Art of Scientific Computing. Cambridge University Press, 1992. 3, 4

[RR95] Robinson J. A., REn M. S.: Data-dependent sampling of two-dimensional signals. Multidimensional Systems and Signal Processing 6 (1995), 89-111. 5

[RVZ08] Romeiro F., VAsilyeV Y., Zickler T.: Passive reflectometry. In Proceedings of the 10th European Conference on Computer Vision: Part IV (2008), ECCV '08, pp. 859-872. 3

[RWS*11] Ren P., WANG J., SNyder J., TONG X., GuO B.: Pocket reflectometry. In ACM SIGGRAPH 2011 papers (2011), SIGGRAPH '11, pp. 45:1-45:10. 2

[SHGW13] Schick E., Herbort S., Grumpe A., Wöhler C.: Single view single light multispectral object segmentation. In WSCG (2013), pp. 171-178. 2

[SSK03] Sattler M., Sarlette R., Klein R.: Efficient and realistic visualization of cloth. In Eurographics Symposium on Rendering (2003), pp. 167-178. 3

[War92] WARD G.: Measuring and modeling anisotropic reflection. Computer Graphics 26, 2 (July 1992), 265 - 272. 2

[WZT*08] Wang J., Zhao S., Tong X., SNyder J., Guo B.: Modeling anisotropic surface reflectance with example-based microfacet synthesis. ACM Tr. Graph. 27, 3 (2008), 41:1-41:9. 4 